\title{
Definitive Chemoradiotherapy Versus Surgery Followed by Adjuvant Radiotherapy in Resectable Stage III/IV Hypopharyngeal Cancer
}

\section{Jun Won Kim, MD ${ }^{1}$ \\ Mi Sun Kim, MD² \\ Se-Heon Kim, MD, PhD ${ }^{3}$ \\ Joo Hang Kim, MD, $\mathrm{PhD}{ }^{4}$ \\ Chang Geol Lee, MD, PhD² \\ Gwi Eon Kim, MD, PhD² \\ Ki Chang Keum, MD, PhD²}

\section{Purpose}

The purpose of this study is to compare the treatment outcomes for locally advanced resectable hypopharyngeal cancer between organ-preserving chemoradiotherapy (CRT) and surgery followed by radiotherapy (SRT).

\section{Materials and Methods}

We reviewed 91 patients with stage III/IV hypopharyngeal squamous cell carcinoma treated with radiotherapy (RT). In the CRT group ( $n=34), 18$ patients were treated with concurrent CRT and 16 patients with induction chemotherapy plus concurrent CRT. In the SRT group $(n=57)$, six patients were treated with total laryngopharyngectomy, 34 patients with total laryngectomy (TL) and partial pharyngectomy (PP), and 17 patients with PP, which were followed by adjuvant radiotherapy $(n=41)$ or CRT $(n=16)$. The median RT dose was 70 Gy for CRT and 59.4 Gy for SRT.

\section{Results}

Five-year local control (84.1\% vs. 90.9\%), and disease-free survival (DFS, $51.0 \%$ vs. $52.7 \%$ ) and overall survival (OS, $58.6 \%$ vs. $56.6 \%$ ) showed no significant difference between the CRT and SRT groups. The functional larynx-preservation rate was higher in the CRT group (88.2\% vs. 29.8\%). Treatment-related toxicity, requiring surgical intervention, occurred more frequently in the SRT group ( $37 \%$ vs. $12 \%$ ). In the SRT group, TL resulted in a significantly higher DFS than larynx-sparing surgery ( $63.9 \%$ vs. $26.5 \%, p=0.027)$. Treatment outcome of the SRT group improved when only patients with TL were considered $(n=40)$; however, 5 -year OS $(67.1 \%$ vs. $58.6 \%, p=0.830)$ and DFS $(63.9 \%$ vs. $51.0 \%, p=0.490)$ did not improve significantly when compared to the CRT group.

\section{Conclusion}

Organ preserving CRT provided a treatment outcome that is comparable to SRT for locally advanced hypopharyngeal cancer, while offering an opportunity for functional larynx-preservation and reduced treatment-related toxicity.

Received December 1, 2014

Accepted January 17, 2015

Published Online March 13, 2015

*Abstract of this work was accepted for the 55th Annual Meeting of the American Society for Radiation Oncology (ASTRO), Atlanta, Georgia, September 2013.

\section{Key words}

Hypopharyngeal neoplasms, Chemoradiotherapy,

Adjuvant radiotherapy, Treatment outcome, Organ preservation

\section{Introduction}

Squamous cell carcinoma (SCC) of the hypopharynx is rare, consisting of 3\%-5\% of all head and neck SCC cases [1]. Hypopharynx is characterized with thin mucosal lining
$(<1 \mathrm{~cm})$ providing minimal barrier with propensity to submucosal spread. A rich lymphatic network of hypopharynx results in frequent lymph node metastases: ipsilateral nodal metastasis in 60\%-80\% and contralateral occult nodal metastasis in up to $40 \%$ [2] of patients at the time of diagnosis. Stage III or IV in $70 \%-85 \%$ patients are reported in large 
series, and 5-year survival is only 15\%-45\% [3].

The preservation of speech and swallowing function in the treatment of laryngeal and hypopharyngeal cancer is important. In early-stage disease, both surgery and radiotherapy (RT) are treatment options that preserve the organ and function $[3,4]$. For locally advanced hypopharyngeal cancer, traditional approach was laryngopharyngectomy and pharyngeal reconstruction with or without adjuvant RT, resulting in a loss of natural voice production. Addition of chemotherapy to RT alone in advanced cancer improved the outcome compared to surgery plus RT, while allowing larynx preservation [5]. However, no clear guidelines specific to hypopharyngeal cancer are available. Most evidences on laryngeal preservation are from laryngeal cancer or mixed head and neck cancer studies. A few studies reported treatment results dedicated to hypopharyngeal cancer, albeit of mixed population, including early stage cancers and advanced unresectable cancers [6].

We reviewed the treatment outcome of locally advanced resectable SCC of the hypopharynx, with comparative analysis between organ-preserving chemoradiotherapy (CRT) and radical surgery followed by adjuvant RT.

\section{Materials and Methods}

\section{Patients}

A total of 136 consecutive patients, who received definitive or adjuvant RT for stage III or IV SCC of the hypopharynx between January 2002 and June 2012, were reviewed. All patients underwent pretreatment evaluation, including a complete history and physical examination, head and neck examination with laryngoscopy, and computed tomography (CT) and magnetic resonance imaging of the head and neck region. All patients were staged in accordance to the 2010 American Joint Committee on Cancer (AJCC) TNM staging system (seventh edition). Patients treated with definitive RT were staged clinically, while both clinical and pathologic stages were reported in postoperative cases. Patients having T1N1 disease $(n=3)$, distant metastasis $(n=3)$, double primary tumor $(\mathrm{n}=11)$, unresectable disease or compromised speech or swallowing functions at presentation $(n=4)$, treated with RT alone $(n=2)$, incomplete RT $(n=3)$, re-irradiation $(n=2)$, and treated with trans-oral robotic surgery $(n=17)$ were excluded, leaving 91 patients for analysis. The study protocol conforms to the Declaration of Helsinki of 1975 as revised in 1983 and was approved by our Institutional Review Board.

\section{Treatment}

Among 91 patients, 57 patients were treated with upfront surgery followed by adjuvant RT (SRT group), and 34 patients were treated with definitive chemoradiotherapy (CRT group). Decisions to undergo CRT were based upon age, medical co-morbidities, and patients' preferences. The SRT group included radical surgery followed by RT $(n=41)$ or concurrent CRT $(n=16)$. Types of surgery included total laryngopharyngectomy $(\mathrm{n}=6)$, total laryngectomy $(\mathrm{TL})$ and partial pharyngectomy (PP) $(\mathrm{n}=34)$, and partial laryngectomy with PP $(\mathrm{n}=17)$. The CRT group included patients treated with concurrent CRT $(\mathrm{n}=18)$ and induction chemotherapy, followed by concurrent CRT ( $\mathrm{n}=16)$. Concurrent chemotherapy regimens included weekly DDP (cisplatin $40 \mathrm{mg} / \mathrm{m}^{2}$ ), weekly FP (5-fluorouracil [5-FU] $750 \mathrm{mg} / \mathrm{m}^{2}$, cisplatin 20 $\left.\mathrm{mg} / \mathrm{m}^{2}\right)$, and TPF $\left(5-F U ~ 750 \mathrm{mg} / \mathrm{m}^{2}\right.$, taxotere $70 \mathrm{mg} / \mathrm{m}^{2}$, cisplatin $75 \mathrm{mg} / \mathrm{m}^{2}$ ) every 3 weeks. Induction chemotherapy regimen consisted of FP every 3 weeks for 3 cycles, or TPF every 3 weeks for 2 cycles. All patients underwent CT-based RT planning with either 3-dimensional conformal RT or intensity-modulated radiotherapy (IMRT). The median RT dose was 59.4 Gy (range, 50.4 to $70.2 \mathrm{~Gy}$ ) for the SRT group and $70 \mathrm{~Gy}$ (range, 59.4 to $72 \mathrm{~Gy}$ ) for the CRT group. For the SRT group, neck dissection was performed for both sides of the neck. Radical neck dissection or modified neck dissection was performed for the involved neck and lateral neck dissection (selective neck dissection in 2 patients) for the clinically uninvolved neck. For the CRT group, both sides of the neck were included in the RT field. Grossly involved lymph nodes were treated with 66-70 Gy, involved cervical nodal stations with 60-63 Gy, and uninvolved nodal stations with 50-54 Gy. Treatment-related toxicities were reported in accordance to the Common Terminology Criteria for Adverse Events (CTCAE) ver. 3.0. CTCAE grade 4 voice alterations or dysphagia after treatment was considered loss of laryngeal function.

\section{Statistical analysis}

The two groups were compared by a chi-square test to detect the differences in proportion. Survival was calculated from the start of treatment until death or to the most recent follow-up date. Survival curves were plotted using a KaplanMeier method. Survival curves were compared using a logrank test for a univariate analysis. The Cox proportional hazards model was used to assess independent predictors for survival. A p-value of less than 0.05 was regarded as statistically significant. 
Table 1. Patient characteristics

\begin{tabular}{|c|c|c|c|c|c|}
\hline Characteristic & & Total $(n=91)$ & SRT $(n=57)$ & CRT (n=34) & p-value \\
\hline Median age (range, yr) & & $64(36-80)$ & $64(39-81)$ & $65.5(37-81)$ & \\
\hline \multirow[t]{2}{*}{ Sex } & Male & $86(94.5)$ & $56(98.2)$ & $30(88.2)$ & \multirow[t]{2}{*}{0.043} \\
\hline & Female & $5(5.5)$ & $1(1.8)$ & $4(11.8)$ & \\
\hline \multirow[t]{3}{*}{ Subsite } & PS & $73(80.2)$ & $48(84.2)$ & $25(73.5)$ & \multirow[t]{3}{*}{0.401} \\
\hline & PW & $13(14.3)$ & $6(10.5)$ & $7(20.6)$ & \\
\hline & PC & $5(5.5)$ & $3(5.3)$ & $2(5.9)$ & \\
\hline \multirow[t]{4}{*}{ cT stage } & cT1 & $8(8.8)$ & $6(10.5)$ & $2(5.9)$ & \multirow[t]{4}{*}{0.342} \\
\hline & cT2 & $26(28.6)$ & $17(29.8)$ & $9(26.5)$ & \\
\hline & cT3 & $27(29.7)$ & $19(33.3)$ & $8(23.5)$ & \\
\hline & cT4a & $30(33.0)$ & $15(26.3)$ & $15(44.1)$ & \\
\hline \multirow[t]{4}{*}{ cN stage } & cN0 & $12(13.2)$ & $6(10.5)$ & $6(17.6)$ & \multirow[t]{4}{*}{0.618} \\
\hline & $\mathrm{cN} 1$ & $14(15.4)$ & $8(14.0)$ & $6(17.6)$ & \\
\hline & $\mathrm{cN} 2$ & $60(65.9)$ & $39(68.4)$ & $21(61.8)$ & \\
\hline & $\mathrm{cN} 3$ & $5(5.5)$ & $4(7.0)$ & $1(2.9)$ & \\
\hline \multirow[t]{3}{*}{ Stage } & III & $17(18.7)$ & $11(19.3)$ & $6(17.6)$ & \multirow[t]{3}{*}{0.629} \\
\hline & IV a & $68(74.7)$ & $42(73.7)$ & $27(79.4)$ & \\
\hline & $\mathrm{IV} \mathrm{b}$ & $6(6.6)$ & $4(7.0)$ & $1(2.9)$ & \\
\hline \multirow[t]{2}{*}{ RT } & 3DCRT & $73(80.2)$ & $51(89.5)$ & $22(64.7)$ & \multirow[t]{2}{*}{0.004} \\
\hline & IMRT & $18(19.8)$ & $6(10.5)$ & $12(35.3)$ & \\
\hline
\end{tabular}

Values are presented as number $(\%)$ unless otherwise indicated. SRT, surgery plus radiotherapy; CRT, chemoradiotherapy; PS, pyriform sinus; PW, pharyngeal wall; PC, postcricoid; cT, clinical T stage; 3DCRT, 3D conformal radiotherapy; IMRT, intensity-modulated radiotherapy.

\section{Results}

\section{Patient characteristics}

Table 1 shows patient and tumor characteristics. The median age was 64 years (range, 36 to 80 years), and male were predominant $(94.5 \%)$. Pyriform sinus $(80.2 \%)$ was the most common subsite of the hypopharyngeal cancer, followed by pharyngeal wall $(14.3 \%)$ and post-cricoid area $(5.5 \%)$. Fifty-seven patients $(62.7 \%)$ had T3-T4a tumors and 65 patients $(71.4 \%)$ had N2-3 nodal disease. Early disease not requiring total laryngectomy (T1-2N0, T1N1) and unresectable primary tumor (T4b) were excluded in this study.

\section{Survival and prognostic factors}

With the median follow-up of 50 months (range, 5 to 141 months), 5-year estimated local failure-free survival (LFFS), locoregional failure-free survival (LRFFS), distant metastasis-free survival (DMFS), disease-free survival (DFS), and overall survival (OS) of all patients ( $\mathrm{n}=91)$ were $88.3 \%, 76.9 \%$, $83.3 \%, 52.1 \%$, and $57.4 \%$, respectively. In a univariate analysis, age $\leq 60$ years (vs. $>60$ ) showed a significant correlation with improved OS $(\mathrm{p}=0.049)$ while sex, subsite, stage, treat- ment group, and RT modality showed no significant correlation. Age $\leq 60$ also showed a trend towards improved DFS $(\mathrm{p}=0.076)$ (Table 2).

\section{Outcome comparison by treatment groups}

Clinical features, including subsite, $\mathrm{T}$ stage, $\mathrm{N}$ stage, and overall stage, were well balanced between the two treatment groups, except for the proportion of female patients $(1.8 \% \mathrm{vs}$. $11.8 \%, \mathrm{p}=0.043)$ and use of IMRT $(10.5 \%$ vs. $35.3 \%, \mathrm{p}=0.004)$ (Table 1). Five-year DFS (52.7\% vs. 51.0\%, p=0.991) and OS (56.6\% vs. $58.6 \%, \mathrm{p}=0.713$ ) showed no significant difference between the SRT and CRT groups (Fig. 1A and B). LFFS (90.9\% vs. 84.1\%, p=0.677), LRFFS (78.8\% vs. 73.7\%, p=0.893), and DMFS ( $80.5 \%$ vs. $88.0 \%, \mathrm{p}=0.507)$ also showed no significant differences.

During the follow-up, treatment failure occurred in 21 patients (36.8\%) in the SRT group and 12 patients in the CRT group (35.3\%). The first sites of failure were three local, five regional, three locoregional, eight distant, one local and distant, and one regional and distant in the SRT group, and five local, three regional, and four distant in the CRT group. Within the SRT group, one local, two locoregional, four regional, and four distant failures occurred among the 40 patients who received total laryngectomy (27.5\% failure), 
Table 2. Univariate analysis for DFS and OS

\begin{tabular}{|c|c|c|c|c|c|c|c|}
\hline \multirow{2}{*}{ Variable } & \multirow{2}{*}{ No. of patients $(\%)$} & \multicolumn{3}{|c|}{ DFS } & \multicolumn{3}{|c|}{ OS } \\
\hline & & 5-Yr DFS (\%) & $95 \% \mathrm{CI}$ & p-value & $5-\operatorname{Yr}$ OS $(\%)$ & $95 \% \mathrm{CI}$ & p-value \\
\hline \multicolumn{8}{|l|}{ Age (yr) } \\
\hline$\leq 60$ & $36(40)$ & 56.9 & $40.2-73.6$ & 0.076 & 62.7 & $46.4-79.0$ & 0.049 \\
\hline$>60$ & $55(60)$ & 49.1 & $35.4-62.8$ & & 54.2 & $40.5-67.9$ & \\
\hline \multicolumn{8}{|l|}{ Sex } \\
\hline Female & $5(6)$ & 80.0 & 44.9-115.1 & 0.396 & 75.0 & $32.5-117.5$ & 0.260 \\
\hline Male & $86(94)$ & 50.3 & $39.3-61.3$ & & 55.0 & $44.0-66.0$ & \\
\hline \multicolumn{8}{|l|}{ Subsite } \\
\hline PS & $73(80)$ & 54.5 & $42.7-66.3$ & 0.606 & 60.2 & $48.6-71.8$ & 0.887 \\
\hline $\mathrm{PW} / \mathrm{PC}$ & $18(20)$ & 42.9 & $19.2-66.6$ & & 46.2 & $21.5-70.9$ & \\
\hline \multicolumn{8}{|l|}{ T stage } \\
\hline T1-2 & $34(37)$ & 57.8 & $40.7-74.9$ & 0.539 & 60.0 & 42.9-77.1 & 0.641 \\
\hline T3-4 & $57(63)$ & 48.8 & $35.3-62.3$ & & 56.2 & $42.7-69.7$ & \\
\hline \multicolumn{8}{|l|}{$\mathrm{N}$ stage } \\
\hline N0-1 & $26(29)$ & 52.6 & $33.0-72.2$ & 0.652 & 59.3 & $39.5-79.1$ & 0.517 \\
\hline $\mathrm{N} 2-3$ & $65(71)$ & 51.9 & $39.2-64.6$ & & 56.6 & $44.1-69.1$ & \\
\hline \multicolumn{8}{|l|}{ Treatment } \\
\hline SRT & $57(63)$ & 52.7 & $39.4-66.0$ & 0.991 & 56.6 & $43.5-69.7$ & 0.713 \\
\hline CRT & $34(37)$ & 51.0 & $33.4-68.6$ & & 58.6 & $41.0-76.2$ & \\
\hline \multicolumn{8}{|l|}{ RT } \\
\hline IMRT & $18(20)$ & 35.7 & $12.2-59.2$ & 0.116 & 53.6 & $27.1-80.0$ & 0.873 \\
\hline 3DCRT & $73(80)$ & 56.3 & $44.5-68.1$ & & 57.9 & 46.3-69.5 & \\
\hline
\end{tabular}

DFS, disease-free survival; OS, overall survival; CI, confidence interval; PS, pyriform sinus; PW, pharyngeal wall; PC, postcricoid; SRT, surgery plus radiotherapy; CRT, chemoradiotherapy; RT, radiotherapy; IMRT, intensity modulated radiotherapy; 3DCRT, 3 dimensional conformal radiotherapy.

and two local, one locoregional, one regional, one local and distant, one regional and distant, four distant failures occurred among the 17 patients who received larynx-sparing surgery (58.8\% failure). Five-year DFS was significantly higher after total laryngectomy than after larynx-sparing surgery (63.9\% vs. $26.5 \%, \mathrm{p}=0.027)$, while total laryngectomy showed a trend towards improved OS compared with larynx-preservation surgery $(67.1 \%$ vs. $31.8 \%, \mathrm{p}=0.094)$ (Fig. 1C and D).

For a subgroup analysis, patients who received total laryngectomy were selected from the SRT group (the SRT/TL group, $n=40$ ) and their treatment outcome was compared with the outcome of the CRT group $(n=34)$. Survival parameters for the SRT/TL group improved when patients who received larynx-sparing surgery were excluded from the subgroup analysis. However, no significant difference was seen in 5-year DFS (63.9\% vs. 51.0\%, p=0.490), OS (67.1\% vs. $58.6 \%, \mathrm{p}=0.830)$, LFFS (90.9\% vs. $84.1 \%, \mathrm{p}=0.677)$, LRFFS $(83.1 \%$ vs. $73.7 \%, \mathrm{p}=0.467)$, and DMFS $(89.7 \%$ vs. $88.0 \%$, $\mathrm{p}=0.732)$ between the SRT / TL and CRT groups.

\section{Outcome of salvage treatment}

Table 3 shows the outcome of salvage treatment among patients who had experienced local and/ or regional failures. Among the 13 patients in the SRT group, only two patients received surgical resection of the recurrent primary tumor or neck nodes, and eight patients were salvaged with chemotherapy or re-irradiation. All patients died of disease progression (survival after recurrence, 1 to 10 months) except for the two patients who were salvaged successfully through re-irradiation with chemotherapy and neck dissection (survival after recurrence, 7 to 24 months). Among eight failures in the CRT group, seven patients were salvaged with dissection of the primary tumor or recurrent cervical lymph nodes. Among the seven patients treated with surgery, three were alive without disease at the time of analysis (survival after recurrence, 5 to 48 months) and three died of intercurrent death without evidence of recurrence (survival after recurrence, 4 to 23 months). 
A
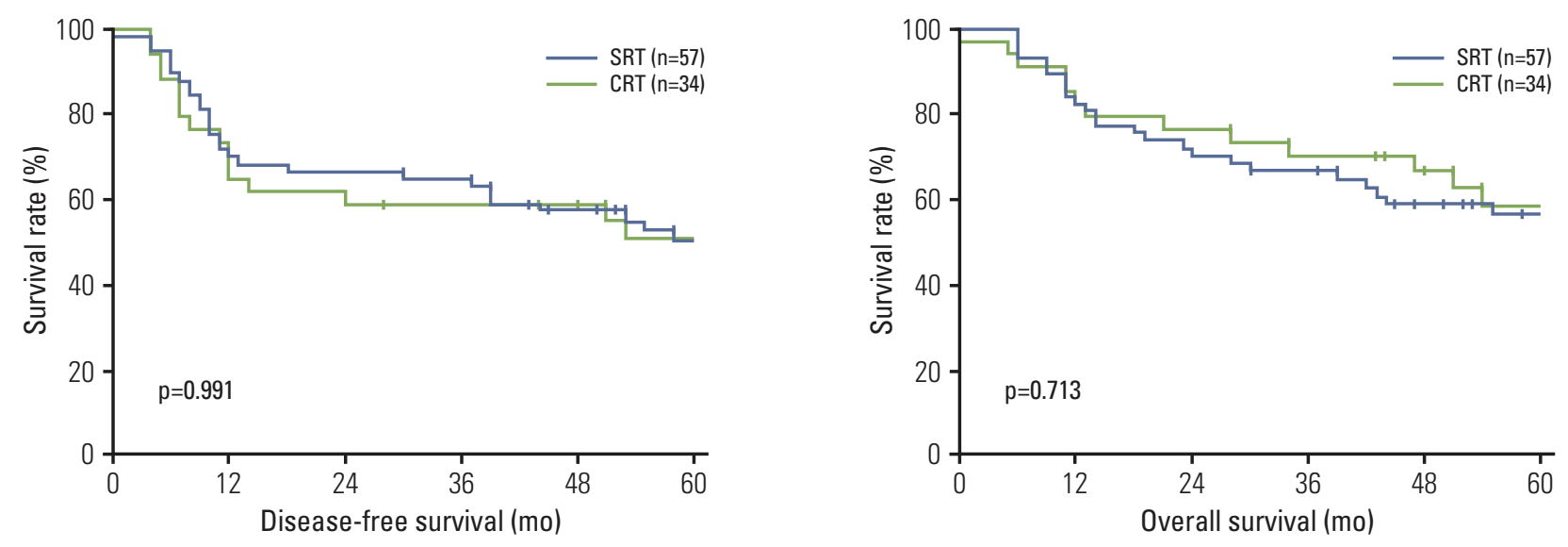

C
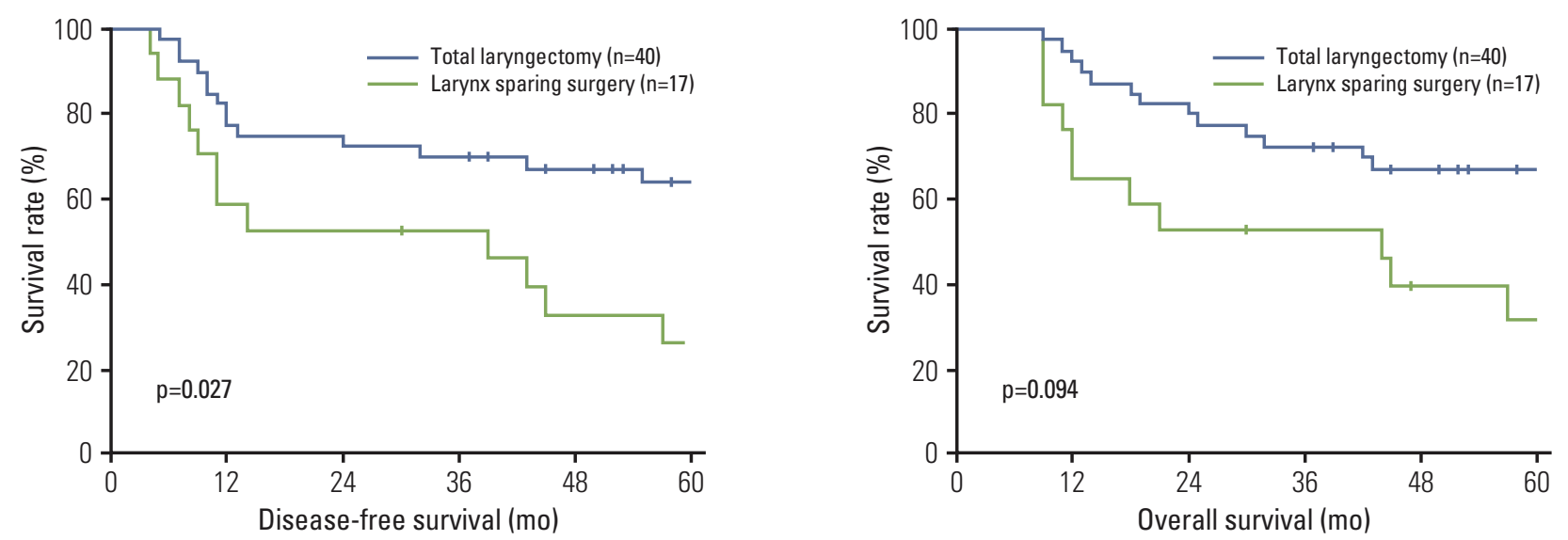

Fig. 1. Comparison of 5-year disease-free survival (A) and 5-year overall survival (B) between the surgery followed by radiotherapy (SRT) and chemoradiotherapy (CRT) groups; comparison of 5-year disease-free survival (C) and 5-year overall survival (D) between total laryngectomy and larynx-sparing surgery in the SRT group.

\section{Toxicity and laryngeal-function preservation rate}

Treatment-related toxicities requiring surgical intervention are listed in Table 4. Wound infection and flap failure are immediate surgical complications, while esophagopharyngeal stenosis, mucocutaneous fistula, and stoma stenosis are late complications. Treatment-related toxicities were more common in the SRT group (37\% vs. 12\%). Table 5 shows toxicities in accordance to CTCAE ver. 3.0 after combined treatment, and grade 4 in voice alteration or dysphagia was considered loss of laryngeal function. Among the 57 patients in the SRT group, 17 retained functional larynx by avoiding total laryngectomy, allowing laryngeal function preservation rate of $29.8 \%$. Among the 34 patients in the CRT group, 30 patients retained a functional larynx (88.2\%). Loss of laryngeal function in four patients in the CRT group was due to local recurrence: three patients after total laryngectomy and one patient from wound infection after salvage partial laryngectomy.

\section{Discussion}

Selecting the optimal treatment strategy for locally advanced hypopharyngeal cancer needs to take into account the extent of the tumor, status of laryngeal function, and 
Table 3. Failure pattern and outcome of salvage treatment

\begin{tabular}{|c|c|c|c|c|c|c|c|c|c|c|}
\hline $\begin{array}{l}\text { Patient } \\
\text { No. }\end{array}$ & $\begin{array}{l}\text { Age (yr) } \\
\text { /Sex }\end{array}$ & Subsite & Stage & Treatment & $\begin{array}{l}\text { Failure } \\
\text { pattern }\end{array}$ & $\begin{array}{l}\text { Stage at } \\
\text { failure }\end{array}$ & $\begin{array}{l}\text { Salvage } \\
\text { treatment }\end{array}$ & $\begin{array}{l}\text { Salvage } \\
\text { response }\end{array}$ & $\begin{array}{l}\text { Current } \\
\text { status }\end{array}$ & $\begin{array}{l}\text { Survival } \\
\text { after } \\
\text { recurrence } \\
\text { (mo) }\end{array}$ \\
\hline 1 & $70 / \mathrm{M}$ & PS & pT1N2c & $\mathrm{P} / \mathrm{P}+\mathrm{RT}$ & $R \& D$ & N2cM1 & CTx & PD & DOD & 1 \\
\hline 2 & $63 / \mathrm{M}$ & PW & pT2N2c & $\mathrm{P} / \mathrm{P}+\mathrm{RT}$ & $\mathrm{R}$ & N1 (CA invas) & None & $\mathrm{n} / \mathrm{a}$ & DOD & 9 \\
\hline 3 & $50 / \mathrm{M}$ & PS & pT4aN0 & $\mathrm{P} / \mathrm{P}+\mathrm{RT}$ & $\mathrm{L}$ & T4aN0 & Excision & PD & DOD & 10 \\
\hline 4 & $65 / \mathrm{M}$ & PW & pT2N2b & $\mathrm{P} / \mathrm{P}+\mathrm{RT}$ & L\& R & T3N1 & None (conservative) & e) $n / a$ & DOD & 2 \\
\hline 5 & $73 / \mathrm{M}$ & PW & pT3N1 & $\mathrm{P} / \mathrm{P}+\mathrm{RT}$ & L \& D & T4aN0M1 & CTx & PD & DOD & 4 \\
\hline 6 & $54 / \mathrm{M}$ & PW & pT2N2b & $\mathrm{P} / \mathrm{P}+\mathrm{RT}$ & $\mathrm{L}$ & T3N0 & CCRT & CR & AWOD & 24 \\
\hline 7 & $55 / \mathrm{M}$ & PS & pT4aN2c & $\mathrm{T} / \mathrm{L}+\mathrm{CCRT}$ & $\mathrm{R}$ & N1 & None (refused) & $\mathrm{n} / \mathrm{a}$ & DOD & 4 \\
\hline 8 & $47 / \mathrm{M}$ & PS & pT4aN2c & $\mathrm{T} / \mathrm{L}+\mathrm{RT}$ & $\mathrm{R}$ & $\mathrm{N} 2 \mathrm{~b}$ & CCRT & PD & DOD & 10 \\
\hline 9 & $65 / \mathrm{M}$ & PS & pT4aN3 & $\mathrm{T} / \mathrm{L}+\mathrm{CCRT}$ & $L \& R$ & T4aN2b & CTx & PD & DOD & 4 \\
\hline 10 & $63 / \mathrm{M}$ & PS & pT4aN2b & $\mathrm{T} / \mathrm{L}+\mathrm{RT}$ & $\mathrm{R}$ & $\mathrm{N} 2 \mathrm{c}$ & CTx & PD & DOD & 7 \\
\hline 11 & $72 / \mathrm{M}$ & PS & pT2N1 & $\mathrm{T} / \mathrm{L}+\mathrm{CCRT}$ & $L \& R$ & T4aN2c & CCRT & PD & DOD & 4 \\
\hline 12 & $54 / \mathrm{M}$ & PS & pT4aN1 & $\mathrm{T} / \mathrm{L}+\mathrm{RT}$ & $\mathrm{R}$ & $\mathrm{N} 2 \mathrm{c}$ & Neck dissection & NED & AWOD & 7 \\
\hline 13 & $57 / \mathrm{M}$ & PS & pT4aN2b & $\mathrm{T} / \mathrm{L}+\mathrm{CCRT}$ & L & T4aN0 & CTx & PD & DOD & 5 \\
\hline 14 & $66 / \mathrm{M}$ & PS & cT3N0 & Induction+CCRT & $\mathrm{L}$ & T4a & None & $\mathrm{n} / \mathrm{a}$ & DOD & 0 \\
\hline 15 & $66 / \mathrm{M}$ & PS & cT4aN2b & Induction+CCRT & $\mathrm{R}$ & $\mathrm{N} 2 \mathrm{~b}$ & Neck dissection & PD & DOD & 9 \\
\hline 16 & $77 / \mathrm{M}$ & PS & cT4aN2b & Induction+CCRT & $\mathrm{L}$ & T4N0 & Transoral P/P & NED & DOOC & 4 \\
\hline 17 & $63 / \mathrm{M}$ & PS & cT1N2b & Induction+CCRT & $\mathrm{R}$ & N1 (CA invas) & ) Neck dissection & NED & DOOC & 17 \\
\hline 18 & $40 / \mathrm{M}$ & PC & cT4aN0 & CCRT & L & T4a & TLP & NED & DOOC & 23 \\
\hline 19 & $68 / \mathrm{M}$ & PS & cT3N1 & CCRT & $\mathrm{L}$ & T4aN0 & TL/PP & NED & AWOD & 48 \\
\hline 20 & $66 / \mathrm{M}$ & PS & cT4aN0 & CCRT & L & T4aN0 & TL/PP & NED & AWOD & 45 \\
\hline 21 & $57 / F$ & PC & $\mathrm{cT} 2 \mathrm{~N} 2 \mathrm{c}$ & CCRT & $\mathrm{R}$ & $\mathrm{N} 2 \mathrm{~b}$ & Neck dissection & NED & AWOD & 5 \\
\hline
\end{tabular}

PS, pyriform sinus; $\mathrm{P}$ / P, partial pharyngectomy; RT, radiotherpy; R, regional; $\mathrm{D}$, distant; $\mathrm{CTx}$, chemotherapy; $\mathrm{PD}$, progression of disease; DOD, died of disease; PW, pharyngeal wall; CA, carotid artery; n/a, not available; L, local; CCRT, concurrent chemoradiotherapy; $\mathrm{CR}$, complete remission; $\mathrm{AWOD}$, alive without disease; T/L, total laryngectomy; NED, no evidence of disease; TLP, total laryngopharyngectomy; DOOC, died of other cause; PC, postcricoid; TL/PP, total laryngectomy \& partial pharyngectomy.

patients' desire for voice preservation. Early stage hypopharyngeal cancer amenable to larynx preservation (most T1N0 and selected T2N0 tumors) may be treated by definitive RT alone or partial laryngopharyngectomy [3,4]. Concurrent CRT or induction chemotherapy, followed by CRT, is recommended for $\mathrm{T} 4 \mathrm{~b}$ or unresectable tumors [7]. It is the spectrum of disease that fall between these early and very advanced disease groups, namely locally advanced resectable hypopharyngeal cancer, that have several treatment options with no clear advantage of one treatment modality over another.

The current study makes a comparison between larynx preserving CRT and radical surgery followed by adjuvant RT, which are both feasible options for locally advanced resectable hypopharyngeal cancer. The 5-year DFS and OS of $52.1 \%$ and $57.4 \%$ for all patients were higher than or at least comparable to the results from previous studies $[1,5,8]$. In this study, the two treatment groups showed comparable DFS and OS. All patients in SRT group underwent adjuvant
RT of median 60 Gy with or without concurrent chemotherapy. It is a well-known fact that postoperative RT or CRT improves tumor control in head and neck cancer patients with high risk features [9]. However, the cost of using adjuvant RT or CRT to improve disease control and survival may be substantial, namely increased treatment-related toxicities and prolongation of the total treatment time. Both treatment groups in the current study were subjected to RT; however, it is difficult to compare treatment-related complications attributable exclusively to RT, since all of the patients received multi-modality treatment. On the other hand, the SRT group experienced higher incidence of surgery-related complications, including wound infection, flap failure, stenosis, and fistula, while patients in the CRT group experienced such complications only when patients were subjected to salvage surgery after a local/regional failure (Table 4).

Another treatment-related complication, which is often overlooked, is a reduced rate of successful salvage treatment 
Table 4. Complications requiring surgical intervention

\begin{tabular}{lcc} 
Variable & SRT $(\mathbf{n = 5 7 )}$ & CRT (n=34) \\
Wound infection & $4(7)$ & $1(3)$ \\
Flap failure & $4(7)$ & 0 \\
Esophageal/pharyngeal stenosis & $8(14)$ & $2(6)$ \\
Pharyngocutaneous fistula & $1(2)$ & 0 \\
Stoma stenosis & $4(7)$ & $1(3)$ \\
\hline
\end{tabular}

Values are presented as number (\%). SRT, surgery plus radiotherapy; CRT, chemoradiotherapy.

Table 5. Laryngeal function after treatment

\begin{tabular}{lccc} 
Variable & CTCAE grade & SRT $(\mathbf{n}=57)$ & CRT $(\mathbf{n}=34)$ \\
\hline Voice alteration & 2 & 3 & 1 \\
& 3 & 1 & 1 \\
Dysphagia & 4 & $40(40)^{\mathrm{a})}$ & $3(3)^{\mathrm{a})}$ \\
& 2 & $35(33)^{\mathrm{a})}$ & 7 \\
& 3 & $9(7)$ & 4 \\
& 4 & 0 & $1(1)^{\mathrm{a}}$ \\
\hline
\end{tabular}

CTCAE, Common Terminology Criteria for Adverse Events; SRT, surgery plus radiotherapy; CRT, chemoradiotherapy. ${ }^{a}$ Numbers in parenthesis are due to total laryngectomy or salvage surgery.

in case of treatment failure. In Fig. 1A and B, DFS curve for SRT is slightly higher than that of CRT, while OS curve for CRT is higher than that of SRT, especially through the later period of follow-up. Such reversal may have been caused by increased number of treatment-related toxicities, higher rate of unsuccessful salvage, or higher incidence of deaths unrelated to disease progression or toxicity during follow-up in the SRT group. Analysis of treatment outcome after local and/or regional failure suggests that CRT allowed more effective salvage treatment in comparison with SRT (Table 3). In the CRT group, six out of eight patients were salvaged through dissection of the primary tumor or recurrent cervical lymph nodes: salvage efforts were successful with three of these patients still alive without disease and three patients died of intercurrent death without evidence of recurrence. Outcome of salvage treatment in the SRT group was less than favorable. Eight of the 13 patients were treated with chemotherapy or re-irradiation, three patients did not receive any treatment, and only two patients received surgical resection of the recurrent tumor. All patients died of disease progression, except for the two patients who were salvaged successfully with re-irradiation and neck dissection. In a clinical setting, most patients who are treated with sur- gery for stage III/IV hypopharyngeal cancer also receive adjuvant RT or CRT. Options for salvage treatment after such intensive combined treatment are limited. These patients have already received radical resection of the primary tumor and / or ipsilateral or bilateral neck dissection, followed by radiation of 60 Gy or higher, which delivers maximum tolerable dose to the critical organs, such as pharyngeal swallowing muscles, spinal cord, and parotid glands. Salvage surgery or re-irradiation is extremely difficult, and chemotherapy alone, a remaining option, is often ineffective against gross recurrent tumor. On the other hand, definitive chemoradiotherapy showed disease control and survival that are comparable to the outcome of surgery plus adjuvant RT or $\mathrm{CRT}$, and in the case of a treatment failure, salvage surgery often results in sustained disease control [5]. In a retrospective series, salvage surgery for failure after chemoradiotherapy has shown acceptable oncologic outcome with no local recurrence after salvage surgery [10].

There are concerns for increased toxicity from intensive chemoradiotherapy, such as impairment of pharyngeal swallowing muscles. Radiation dose to hypopharynx was associated with severe late toxicity in accordance to Radiation Therapy Oncology Group (RTOG) analysis [11], and functional outcome of CRT for head and neck cancer was not so promising, despite improved locoregional control [12]. In unsuccessful organ preservation attempts, failures in function preservation may result from either toxicity from aggressive CRT, extensive tumor infiltration into the organ at the time of diagnosis, or from progression of disease. Patients with functional impairment due to tumor infiltration should be excluded from organ preservation candidates. In this study, patients who had resectable tumor $(<\mathrm{T} 4 \mathrm{~b})$ and intact laryngeal function were included for analysis, and outcome of larynx preserving CRT was compared to the outcome of radical surgery followed by adjuvant RT/CRT. The function preservation rate of $88.2 \%$ in the CRT group with loss of laryngeal function only due to local recurrence or complication of salvage surgery may lead to decisions favoring larynx preserving treatment options.

Previous studies on treatment outcome of head and neck cancer fail to take into account recent advances in treatment modalities and discovery of new prognostic factors. The use of IMRT allowed for a reduction of radiation-induced toxicity, improved local and regional control, and potential for improved survival in hypopharyngeal and other head and neck cancer patients [13]. The most important factors that contribute to morbidity in head and neck cancer patients undergoing intensive CRT are increased risk of dysphagia and aspiration. By more accurate delineation of target volumes and sparing of pharyngeal constrictors and supraglottic larynx, patients treated with IMRT experienced fewer swallowing problems than those treated with conventional 
RT [14]. Treatment deintensification in patients with human papillomavirus (HPV) positive oropharyngeal cancer is gaining interests [15]. A number of studies reported implication of HPV-positive hypopharyngeal cancer in organ preservation therapy $[16,17]$. Further investigations may allow deintensification in HPV-positive hypopharyngeal cancer and reduction of treatment-related toxicities while maintaining treatment outcome.

Efforts have been made to employ less than radical surgery for hypopharyngeal cancer. Partial pharyngectomy and even trans-oral resection of the tumor have been attempted with the purpose of organ preservation $[3,18]$. However, these limited surgery options should be explored with caution, especially in locally advanced cancer of the hypopharynx. In this study, the outcome of patients treated with partial laryngectomy and pharyngectomy was poor compared to the outcome of total laryngectomy. Close and positive resection margin was reported in six $(35.3 \%)$ and five $(29.4 \%)$ of the 17 patients who received larynx-sparing surgery and $11(27.5 \%)$ and six $(15 \%)$ of 40 patients who received total laryngectomy. Local failure occurred in three patients among the 40 who received total laryngectomy $(7.5 \%)$ and in four among the 17 patients who received less than total laryngectomy (23.5\%) (Table 3). Larynx preservation through avoidance of total laryngectomy does not seem to be effective due to its high rate of local failure. The incidence of submucosal extension is up to $58 \%$ [19], and pathological studies showed that extent of submucosal involvement measured from the edge of the tumor ranged $10-20 \mathrm{~mm}$ superiorly and $10-30 \mathrm{~mm}$ inferiorly [20,21], requiring resections margins of $3 \mathrm{~cm}$ inferiorly and $2 \mathrm{~cm}$ both superiorly and laterally. In locally advanced hypopharyngeal cancer, it is difficult to perform laryngeal preservation surgery while achieving adequate resection margins.

One of the limitations in the current study is the inclusion of a heterogeneous group of patients in the CRT group. The CRT group consists of patients treated with concurrent CRT as well as sequential CRT (induction chemotherapy plus RT or CRT). Recent reports on the induction chemotherapy for laryngeal preservation have shown equivalent outcome between sequential CRT and concurrent CRT [22,23]. The number of cases reviewed in the current study is relatively small, albeit only locally advanced resectable hypopharyngeal cancer cases are included for analysis. Disease characteristics of SCC of the hypopharynx are unique, and optimizing combined modality treatment needs to be explored further with a larger cohort of patients. In the current study, patient characteristics were well balanced between the SRT and CRT groups except for the proportion of female patients, and the use of IMRT were higher in the CRT group. In a univariate analysis, female sex and use of IMRT did not result in significant improvement in DFS and
OS (Table 1). Although women have been found to achieve somewhat improved outcomes compared to men, this may in part be due to earlier-stage disease at diagnosis [24]. Moreover, the proportion of female patients $(5.5 \%)$ was too small to cause a significant difference in the treatment outcome in this study. IMRT is superior over conventional RT in reducing radiation induced toxicities; however, its advantage in patient survival is unclear. In a randomized trial comparing IMRT and conventional RT for the treatment of oropharyngeal and hypopharyngeal SCC, IMRT was superior in preventing xerostomia; however, no significant differences were observed in non-xerostomia late toxicities, locoregional control or overall survival between IMRT and conventional RT [25].

\section{Conclusion}

Organ preservation is not to be confused with function preservation. For hypopharyngeal cancer, functions include voice and swallowing, as well as quality of life. In hypopharyngeal cancer with impaired laryngeal function at presentation, laryngopharyngectomy with pharyngeal reconstruction is a preferred option permeating aspiration-free deglutition and prosthetic voice instead of leaving intact but functionless larynx. However, definitive CRT may be preferred in locally advanced resectable hypopharyngeal cancer with intact laryngeal function, providing treatment outcome comparable to surgery plus RT or CRT, while shortening total treatment time and proving a salvage chance in the case of local/regional failure.

\section{Conflicts of Interest}

Conflict of interest relevant to this article was not reported.

\section{Acknowledgments}

This work was supported by a faculty research grant of Yonsei University College of Medicine for 2013 (Grant No. 6-2013-0057). 


\section{References}

1. Hall SF, Groome PA, Irish J, O'Sullivan B. The natural history of patients with squamous cell carcinoma of the hypopharynx. Laryngoscope. 2008;118:1362-71.

2. Buckley JG, MacLennan K. Cervical node metastases in laryngeal and hypopharyngeal cancer: a prospective analysis of prevalence and distribution. Head Neck. 2000;22:380-5.

3. Takes RP, Strojan P, Silver CE, Bradley PJ, Haigentz M Jr, Wolf GT, et al. Current trends in initial management of hypopharyngeal cancer: the declining use of open surgery. Head Neck. 2012;34:270-81.

4. Jones AS. The management of early hypopharyngeal cancer: primary radiotherapy and salvage surgery. Clin Otolaryngol Allied Sci. 1992;17:545-9.

5. Lefebvre JL, Chevalier D, Luboinski B, Kirkpatrick A, Collette L, Sahmoud T. Larynx preservation in pyriform sinus cancer: preliminary results of a European Organization for Research and Treatment of Cancer phase III trial. EORTC Head and Neck Cancer Cooperative Group. J Natl Cancer Inst. 1996;88: 890-9.

6. Chen SW, Tsai MH, Yang SN, Liang JA, Shiau AC, Lin FJ. Hypopharyngeal cancer treatment based on definitive radiotherapy: who is suitable for laryngeal preservation? J Laryngol Otol. 2008;122:506-12.

7. D'Cruz A, Lin T, Anand AK, Atmakusuma D, Calaguas MJ, Chitapanarux I, et al. Consensus recommendations for management of head and neck cancer in Asian countries: a review of international guidelines. Oral Oncol. 2013;49:872-7.

8. Blanchard P, Tao Y, Veresezan O, Lusinchi A, Le Ridant AM, Janot $F$, et al. Definitive radiotherapy for squamous cell carcinoma of the pyriform sinus. Radiother Oncol. 2012;105:232-7.

9. Bernier J, Cooper JS, Pajak TF, van Glabbeke M, Bourhis J, Forastiere A, et al. Defining risk levels in locally advanced head and neck cancers: a comparative analysis of concurrent postoperative radiation plus chemotherapy trials of the EORTC (\#22931) and RTOG (\# 9501). Head Neck. 2005;27: 843-50.

10. Suzuki K, Hayashi R, Ebihara M, Miyazaki M, Shinozaki T, Daiko $\mathrm{H}$, et al. The effectiveness of chemoradiation therapy and salvage surgery for hypopharyngeal squamous cell carcinoma. Jpn J Clin Oncol. 2013;43:1210-7.

11. Machtay M, Moughan J, Farach A, Martin-O'Meara E, Galvin J, Garden AS, et al. Hypopharyngeal dose is associated with severe late toxicity in locally advanced head-and-neck cancer: an RTOG analysis. Int J Radiat Oncol Biol Phys. 2012;84: 983-9.

12. Tulunay-Ugur OE, McClinton C, Young Z, Penagaricano JA, Maddox AM, Vural E. Functional outcomes of chemoradiation in patients with head and neck cancer. Otolaryngol Head Neck Surg. 2013;148:64-8.

13. Al-Mamgani A, Mehilal R, van Rooij PH, Tans L, Sewnaik A, Levendag PC. Toxicity, quality of life, and functional outcomes of 176 hypopharyngeal cancer patients treated by (chemo)radiation: the impact of treatment modality and radiation technique. Laryngoscope. 2012;122:1789-95.

14. Vergeer MR, Doornaert PA, Rietveld DH, Leemans CR, Slotman BJ, Langendijk JA. Intensity-modulated radiotherapy reduces radiation-induced morbidity and improves healthrelated quality of life: results of a nonrandomized prospective study using a standardized follow-up program. Int J Radiat Oncol Biol Phys. 2009;74:1-8.

15. O'Sullivan B, Huang SH, Siu LL, Waldron J, Zhao H, PerezOrdonez B, et al. Deintensification candidate subgroups in human papillomavirus-related oropharyngeal cancer according to minimal risk of distant metastasis. J Clin Oncol. 2013;31: 543-50.

16. Joo YH, Lee YS, Cho KJ, Park JO, Nam IC, Kim CS, et al. Characteristics and prognostic implications of high-risk HPV-associated hypopharyngeal cancers. PLoS One. 2013;8:e78718.

17. Shaughnessy JN, Farghaly H, Wilson L, Redman R, Potts K, Bumpous J, et al. HPV: a factor in organ preservation for locally advanced larynx and hypopharynx cancer? Am J Otolaryngol. 2014;35:19-24.

18. Kuo YL, Chang CF, Chang SY, Chu PY. Partial laryngopharyngectomy in the treatment of squamous cell carcinoma of hypopharynx: analysis of the oncologic results and laryngeal preservation rate. Acta Otolaryngol. 2012;132:1342-6.

19. Ho CM, Ng WF, Lam KH, Wei WJ, Yuen AP. Submucosal tumor extension in hypopharyngeal cancer. Arch Otolaryngol Head Neck Surg. 1997;123:959-65.

20. Harrison DF. Pathology of hypopharyngeal cancer in relation to surgical management. J Laryngol Otol. 1970;84:349-67.

21. Davidge-Pitts KJ, Mannel A. Pharyngolaryngectomy with extrathoracic esophagectomy. Head Neck Surg. 1983;6:571-4.

22. Forastiere AA, Zhang Q, Weber RS, Maor MH, Goepfert H, Pajak TF, et al. Long-term results of RTOG 91-11: a comparison of three nonsurgical treatment strategies to preserve the larynx in patients with locally advanced larynx cancer. J Clin Oncol. 2013;31:845-52.

23. Haddad R, O'Neill A, Rabinowits G, Tishler R, Khuri F, Adkins D, et al. Induction chemotherapy followed by concurrent chemoradiotherapy (sequential chemoradiotherapy) versus concurrent chemoradiotherapy alone in locally advanced head and neck cancer (PARADIGM): a randomised phase 3 trial. Lancet Oncol. 2013;14:257-64.

24. Spector JG, Sessions DG, Emami B, Simpson J, Haughey B, Harvey J, et al. Squamous cell carcinoma of the pyriform sinus: a nonrandomized comparison of therapeutic modalities and long-term results. Laryngoscope. 1995;105(4 Pt 1):397-406.

25. Nutting CM, Morden JP, Harrington KJ, Urbano TG, Bhide SA, Clark C, et al. Parotid-sparing intensity modulated versus conventional radiotherapy in head and neck cancer (PARSPORT): a phase 3 multicentre randomised controlled trial. Lancet Oncol. 2011;12:127-36. 